\title{
A Review on Ruben's Tube as Acoustic Propagator
}

\author{
R. Salim, S. T. Syed Shazali*, S. Hamdan, M. Andrew-Munot and \\ A. M. A. A. M. Mohtar \\ Faculty of Engineering, Universiti Malaysia Sarawak (UNIMAS), \\ Jalan Datuk Mohammad Musa, 94300, Kota Samarahan, Sarawak, Malaysia \\ *E-mail: starmizi@ unimas.my \\ Phone: +(60)82 583296, Fax: +(60)82 583410
}

\begin{abstract}
In 1905, Ruben's Tube was invented by German physicists Heinrich Ruben and Otto Krigar-Menzel. The tube is closed at one end and the other end is attached with a loudspeaker, then filled with flammable gas. With the help of the Ruben's Tube, the standing waves were represented physically by fire. There are no specific standards in which Ruben's Tube is constructed. At certain resonance frequency, the flame produced visual standing wave that varies in height. It has been used in the teaching of acoustical resonance behavior. This article provides review on Ruben's Tube and its operation, as well as some commentaries and drawbacks for each subtopic. Each of the journals that have similar setup have been reviewed to further explain the phenomena.
\end{abstract}

Keywords: Ruben's Tube; standing wave behaviours; nodes and antinodes.

\section{INTRODUCTION}

\section{What is Ruben's Tube?}

Sound propagates through air in the form of pressure waves. However, it is difficult to watch a standing wave with the naked eye. Therefore, it can be visualized using the Ruben's Tube. By passing flammable gas through a long metal tube with a small hole drilled in the top, it is possible to create a line of individual flames. The height of each flame is related to the gas flow rate through the hole beneath it, which can be altered by modifying the gas pressure inside the tube. If this is done by a suitable choice of sound waves, standing waves can be established inside the tube, resulting in a flame pattern. This can then be examined to establish relationships between the sound waves and the gas in which they travel. Figure 1 shows the experiment setup for Ruben's Tube.

\section{Fundamental Theory of Ruben's Tube}

In this experiment, the tube is closed at one end. The number of nodes and antinodes produced are the same. The experiment starts with nodes, ends with antinodes, and the maxima could occur at nodes or antinodes [1].

Many years later, myriad of experiments have been done to prove the above statement. Reference [3] shows that the maxima of flame would occur at antinodes. Then, another experiment had been conducted to show that the maxima of flame would be at antinodes [4], [12]. All of these experiments have raised doubts from many people who had asked for more information from the 'The Physics Teacher' for further clarification 
[13]. Therefore, the experiments explain that under normal conditions, the flame is yellow and tall at nodes, while blue and short [14] at antinodes. Hence, it can be concluded that the flame maxima occurred at pressure nodes. However, under high sound intensities (140-150 dB), variations in pressure occuring inside the tube could reverse the maxima of flame [14].

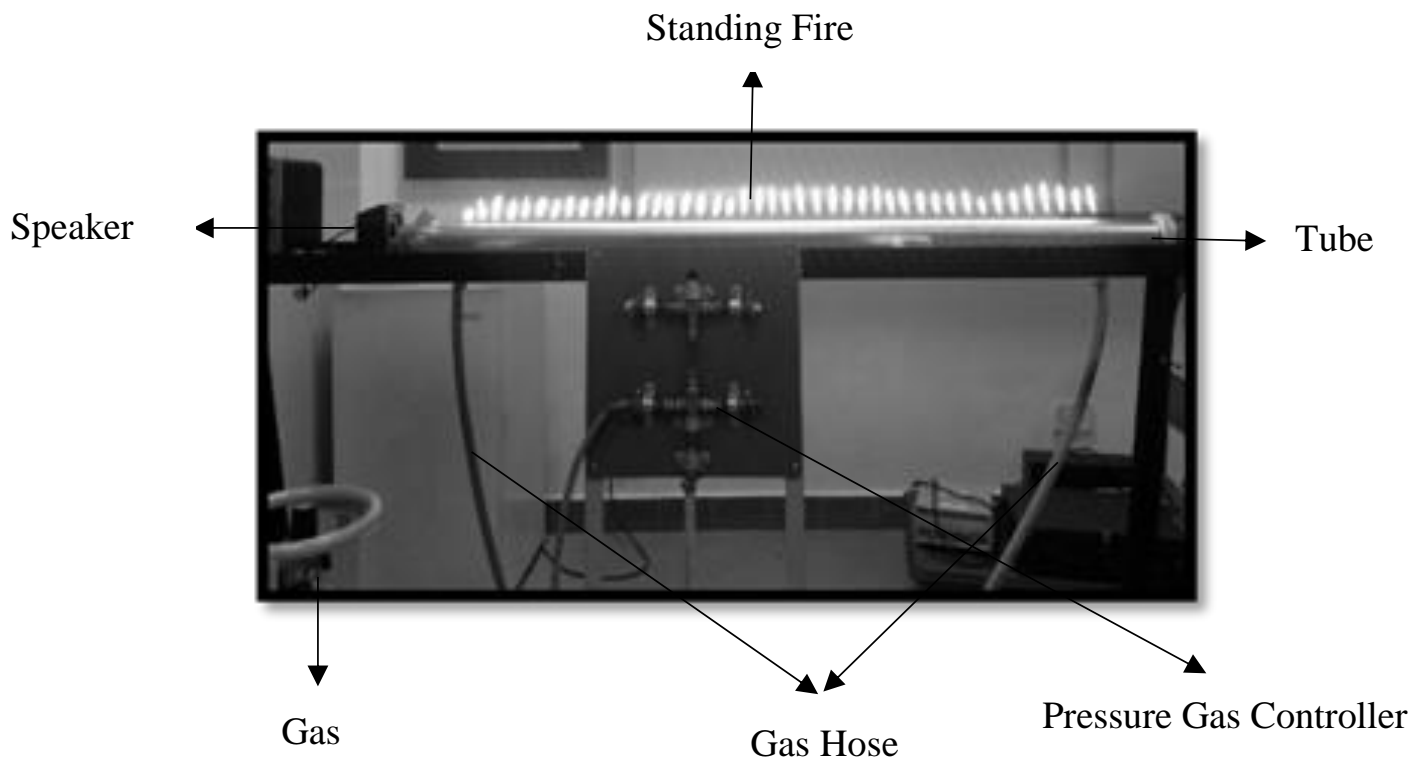

Figure 1. Experiment setup for Ruben's Tube.

There is a journal that also answered the question claiming that the factor of static gas pressure is important to decide the maxima of flame arising [15]. Under normal static gas pressure, the flame maxima occurred at pressure nodes and low static pressure maxima could happen at pressure antinodes. Therefore, this supported the statement of Ruben and Krigar-Menzel where the flame maxima occur at pressure nodes and minima at pressure antinodes. However, the factor of sound intensity and static gas pressure can influence the location of flame maxima.

Reference [9] show that the theory on Bernoulli equation is based upon a simple model that can be used to check the mass flow rate inside the tube. It proved under normal conditions, the maxima occur at pressure nodes based on time-averaged mass flow rate. Nevertheless, increasing the sound level and decreasing the static pressure will reverse the effect in flame height which is the flame maxima at the antinodes and flame minima at the pressure nodes. However, there is an argument which leads to some disagreement on the explanation by claiming that the flame maxima occur at pressure antinodes [5]. Figure 2 show the nodes and antinodes produced during the experiment.

\section{Variation Type of Ruben's Tube}

There are many variations on Ruben's Tube design, with each innovation having its own specific benefits. For example, there are articles on construction of Ruben's Tube in square and circular flames tables [10][11]. The purpose of this design is to show the twodimensional standing wave patterns in different systems. Apart from that, an air track system can be modified to become a flame tube setup [22]. The design has its advantages where space and budget is taken into considerations in constructing Ruben's Tube. 


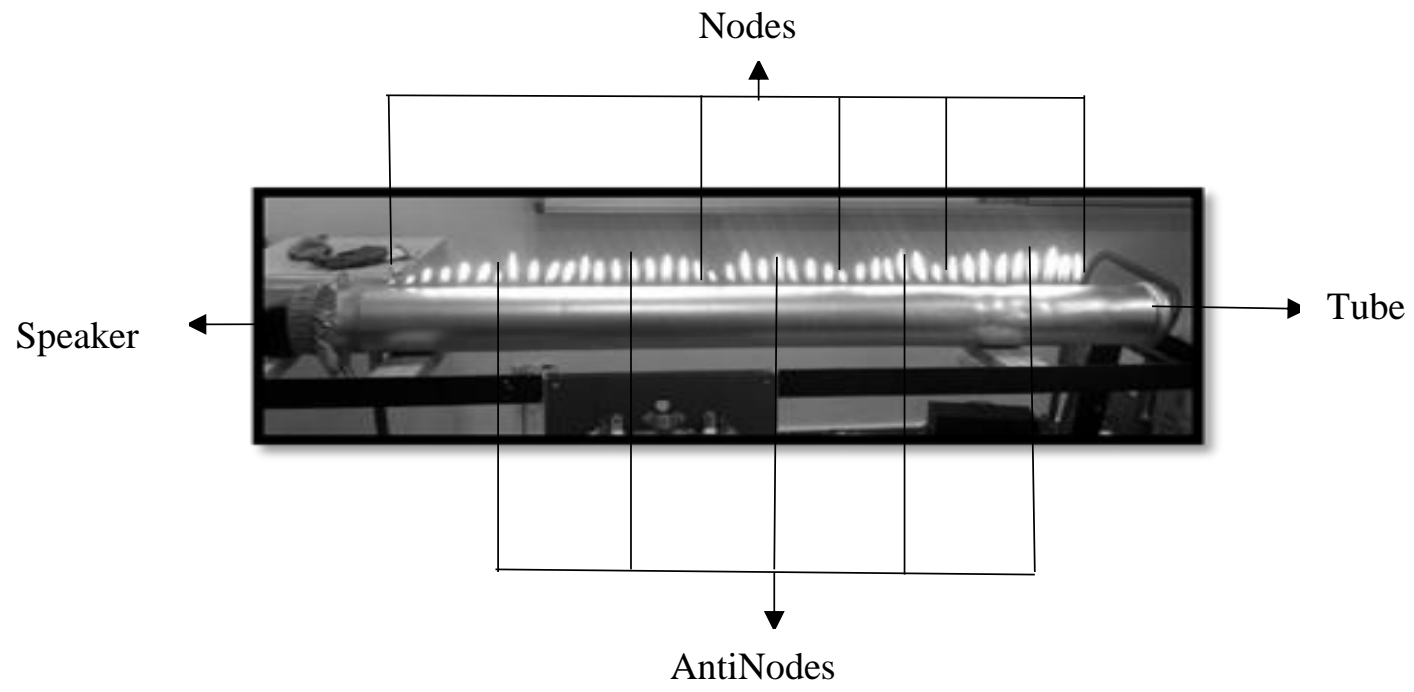

Figure 2 Production of nodes and antinodes.

\section{RELATED WORK}

Previous journals are reviewed to have a better understanding of the Ruben's Tube. Previous works only focused on using numerical software to make the tube as an integrated system, using different types of gas to check the speed of gas and the frequency inside the tube, and using different diameter sizes of the tube to check the flame propagation which significantly affect the occurrence of vortex flow inside the tube.

\section{Ruben's Tube as an Integrated System}

Ruben's Tube can be used altogether as an integrated system. For example, it can be proved by investigating the effects of frequency changes on standing waves that the sound wave is also a pressure wave caused by vibrations occurring inside the tube [19]. This experiment used the same type of gas in entirety: propane gas. Distance between the tube and the loudspeaker diaphragm are constant to prevent errors during the experiment. After several data and calculation has been made, it is shown that the different wavelengths are inversely proportional to different frequencies.

As the frequency increases, the number of antinodes and nodes increases, while wavelength decreases, showing the inverse relationship between wavelength and frequency. In an early experiment, the author used music to watch the movement of the flame. However, it is difficult to get precise result using music because it contains various frequencies that change continuously. Therefore, instead of using music, this experiment used a software that specialises in record and edit sound such as Audacity® to form single sinusoidal harmonic sound from $47.7 \mathrm{~Hz}$ until $334 \mathrm{~Hz}$.

The resonance frequencies were different from the calculated frequency [7]. It suggested that the difference between the resonance frequency during the experiment was due to the size of holes. The size of holes would affect the behaviour of the tube. Smaller and fewer holes decreased the resonance frequency shift. However, the holes cannot be too small or too far apart because they would affect the performance during 
demonstration. In this experiment, MATLAB®-based Graphical User Interface (GUI) was developed to complete the modelled tube as shown in Figure 3. This model consisted of various dimensions such as tube radius, hole spacing, number, size, etc. Also, the presence of the loudspeaker affected the result. This was due to the loudspeaker changing the resonance frequencies and effected the result during the first few harmonics. The loudspeaker itself had its own resonant frequency and introduced a significant damping to the system.

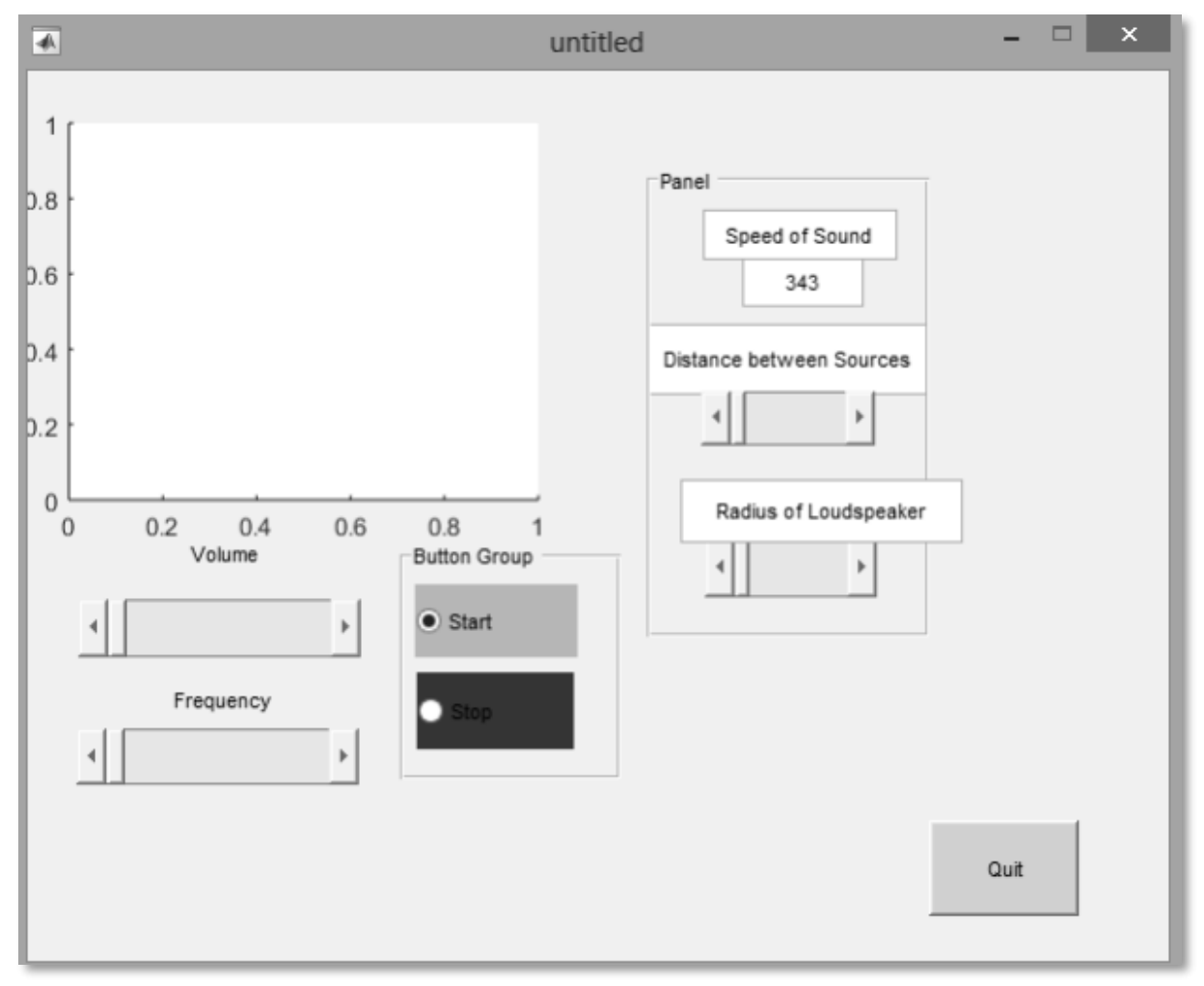

Figure 3 Example of button at layout area for GUI.

Stereoscopic video camera was used to track the motion and follow the signal of a dancer [25]. Also, this project had a small and light wireless sensor system worn by the dancer. Firetrase stands for Fire, Tracking and Sensors. A pyro technician developed a real time software to control each of the ramp of 20 fire projectors height individually [25] for this project. In addition, the authors created a flexible patterns generator program to make and combine different types of complex patterns used to control the movement of a dancer. However, there were difficulties to perform with real fire. Hence, to overcome this problem, the authors combined results from the project with the pitch detection algorithms from the Multimodal Guitar project. As a result, the program could control many types of sound transformations, pre-recorded sound sequences and specialisation concurrently with real flame.

Reference [17] shows direct demonstration of an audio-range line array that can be used to explain the interference of various sources in a laboratory or physical classroom. In this experiment, the authors developed a GUI using LABVIEW@ that can permit real time control, sine wave of user specified frequency, amplitude, steering of the array and phase shading. The GUI permits changes in frequency, the angular shading by phase shading and reduces side lopes through amplitude shading. Apart from that, the 
GUI used a total of eight inexpensive loudspeaker arrays to test the software in an anechoic chamber and in a big classroom. The advantage of this software is it can switch on and off each individual loudspeaker and control the arbitrary phase and amplitude of the program.

Reference [26] shows that numerical simulations were performed to study the effects of pressure waves on the development and stability of flames propagating in tubes. The numerical solution method is high in order in space and time, adaptive mesh refinement provides adequate resolution of flames, acoustic waves, boundary layers and has all the interactions with others. Series of Distorted Tulip Flames (DTFs) and Tulip Flames (TFs) show the influence of tube length scale and aspect ratio for a range of tube sizes. Acoustic properties of the tube and pressure waves has an important role in the flame evolution, especially in the formation and evolution of the series of increasingly wrinkled DTFs [26].

There is an effect of external heat loss on the slow propagation of strong-burning flames inside narrow heat recirculating tubes [8]. This study used a 2-D numerical model for reactive flow, including conjugated heat transfer, over a range of tube diameters spanning the micro-scale (small scale) and the meso-scale (larger scale). Flames in microscale has an extinction limit, while flames in large diameter tubes such as in meso-scale range, usually has a blowout limit. The 2-D model can show the flame shape more efficiently than a 1-D model. It also allows an increase in burning surface area which has more stability to the system. Hence, choosing the right size tube diameter is important to the design of a stable burner optimized for heat transfers to an external heat load.

Upward propagation of a premixed flame in a vertical tube has a very lean mixture of gas [20]. By using a single irreversible Arrhenius reaction model, this experiment underwent a numerical simulation with high activation energy. It showed that the flow might become unstable when the fuel mass fraction was decreased, and together with flame stretch due to strong curvature of the flame tip in narrow tube, might be the reason for the minimum fuel mass fraction.

There are also experimental and numerical investigations that have been carried out to describe methane ignition driven long wall coal mine explosions, which were among the biggest industrial explosions [6]. The research investigated methane combustion with flame propagation in horizontal cylindrical tubes and its effect on tube diameter of different sizes. In the experiment, the authors used horizontal tubes with one end venting to the atmosphere, and the other end to a solid wall. The tube diameter used were between 5 to $71 \mathrm{~cm}$. The outcome proved that maximum laminar flame propagation velocity increases with tube diameter. Also, the 2-D simulations for these tubes were carried out with ANSYS Fluent.

\section{Relationship Between Sound Waves and Sound Pressure}

Ruben's Tube helps to show the relationship between sound waves and sound pressure, which is one of the important objectives in this review paper [18], by doing a demonstration with fire. The experimental setup consists of a tube that is constructed to be airtight, with holes drilled at regular intervals across the length at the top of the tube. There exists a gas line, and one end of the tube is fitted with a diaphragm. When the gas is pumped into the tube, it will then be forced out of the tube through the holes. At this point the gas is ignited to create individual flames at each of the holes. When a frequency is played across the diaphragm, the resulting pressure in the tube causes the flames to vary in height. This experiment uses two types of gas such as air and propane. To calculate 
the speed of gas, the authors make comparisons between their theories and the experiment's results.

Ruben's Tube use to measure different wavelengths is inversely proportional to different frequencies [21]. Calculations were made to predict a wavelength at different frequencies and compare it with the result obtained from the experiment. Therefore, high frequencies will produce shorter wavelengths. This experiment used $300 \mathrm{Hertz}(\mathrm{Hz})$ to $700 \mathrm{~Hz}$ to measure different wavelengths. Three different trials were done to measure the averages of each wavelength. Using percentage error analysis to compare the theoretical with the experiment values achieves the objective of the experiment to prove higher frequencies tend to have small wavelengths. The wavelength can be calculated for different frequencies using the following Eq. (1).

$$
\mathrm{f}=\frac{\mathrm{v}}{\lambda}
$$

where $f$ is frequency, $v$ is for speed of sound and $\lambda$ is wavelength. For this experiment the velocity of sound in gas will be calculated using Eq. (2)

$$
\mathrm{v}=\left(\frac{\mathrm{K}}{\rho}\right)^{\frac{1}{2}}
$$

where $K$ is the modulus of bulk while $\rho$ is density of the gas.

Another article explains the acoustic standing wave behaviour is based on the height of flame produced above the tube Error! Reference source not found.. This research explains the history of Ruben's Tube, from the tube development to its operation. There are also commentaries and other experiments that involved the flame tube itself in literature reviews. Explanations on flame maxima occurrences came from various sources and opinion. Originally, it stated that the flame maxima could occur either at nodes and antinodes [1]. During normal conditions, the flame maxima appear to be at nodes. However, during revised conditions, the flame maxima could occur at antinodes due to the changes in sound intensity and static gas pressure that appear during the experiments.

\section{Comparison of the Size of Tube Diameter}

Differences in tube diameter also affect the performance of flame propagation in the tube. There was an experiment done to investigate the premixed propane and methane-air flames propagating in a tube 1.5-meter-long, with diameters $54 \mathrm{~mm}$ and $94 \mathrm{~mm} \mathrm{[16].} \mathrm{The}$ tubes were open at one end, while the other end was closed with an acoustic damper to remove thermo-acoustic instability inside the tube. This experiment was distinguished by correlating the flame speed and the radius of curvature at the flame tip. The characteristic lengths were then related to the cut-off wavelengths estimated from linear theories and compared to previous results of Michelson-Sivashinsky simulations. The effective radius of curvature was obtained by using the Eq. (3) below.

$$
\frac{2}{\mathrm{Rf}}=\frac{1}{\mathrm{R} 1}+\frac{1}{\mathrm{R} 2}
$$

where $\mathrm{R}_{\mathrm{f}}$ is for radius of curvature, $\mathrm{R}_{1}$ is for large radius and $\mathrm{R}_{2}$ is for small radius. Small diameter tubes and swirl injectors were used for methane and propane/air mixtures [24]. 
By using a $3.6 \mathrm{~mm}$ tube, it showed that a flame can propagate rapidly along the tube axis due to vortex bursting, as shown in larger diameter tubes. Also, the propagation range increases and decreases in relation to air flow rate, and further decreasing can lead to a failure in propagation [24]. When the swirl intensity is increased, the flame propagation range in the equivalent ratio and in the air flow rate widen. Flame propagation for methane gas shift to fuel-lean side while for propane gas it will shift to fuel-rich side. These ranges are narrower than the ones obtained in a $31 \mathrm{~mm}$ tube, which is of a larger diameter.

Furthermore, flames in narrow circular tubes show how the thickness of tube diameter effects the flame propagation inside the tube [15]. The fuel-rich inflammable mixture is blown down the tube, which is open at one end and supported deflagration that sometimes has steady and unsteady flow. The unsteadiness is characterized by periodic extinction and reigniting. There is also a mathematical model that is able to capture both the dynamics and the transitions in parameter space between steady solutions, unsteady solutions and a return to steady solutions.

When a flame passes through a tube that is open at both ends, a self-induced fluctuating pressure or flow field is created in which the flame has to traverse [27]. This experiment uses fuel of propane-air flame and films it while travelling along a $20 \mathrm{~mm}$ internal diameter quartz tube. During the experiment, fluctuations in the flame's progression was observed to increase as the flame propagated, achieving a maximum oscillation amplitude of $\pm 10 \mathrm{~mm}$ at $220 \mathrm{~Hz}$ that decayed as the flame progressed further towards the ends of the tube [27]. The changes of the periodic pressure gradients on the flame shape could be discerned as the unburned reactants push themselves through the products together with the corresponding rapid acceleration of the flame into the unburned mixture. The changes of the flame were observed by capturing the hydrocarbon $\left(\mathrm{CH}^{*}\right)$ and diatomic carbon $\left(\mathrm{C}_{2}\right)$ chemiluminescence using a high-speed colour camera. Hence, the $\mathrm{CH}^{*} / \mathrm{C}_{2}$ ratio was observed to show a lower ratio as the flame was pulled back towards the burned mixture, and a higher ratio when the flame was pushed forward.

Apart from that, there is an experiment to study the effects of both the bend angle and bend position of flame acceleration in pipes [28]. There are three stages for flame acceleration in a pipe bend. For example, the flame speed increases before the bend, increases again after decreasing for a short distance in the bend, decreasing flame speed during reversal of flame in the bend that leads to an additional turbulence which enhances the flame acceleration after the bend. All three stages have a similar case due to the decreasing amplitude of the flame speed in the bend, which increases with a decrease in the bend angle. The flame speeds in the bend were ordered such that $52^{\circ}<90^{\circ}<145^{\circ}$, but the maximum flame speeds in the pipe were in the opposite order [28]. However, both the flame and its speed in the whole pipes increase as the bend's position away from ignition point is increased. There is another type of equation used other than the equation (3) to compare the different sizes of tube diameter. This is due to a combination of mathematical model and the different types of simulation used to make a comparison between the sizes of tube diameter for each review journal.

\section{CONCLUSION}

Many journals and patents have been published to explain the behaviour of sound waves. Prior publications show greater advantages. By using Ruben's Tube as an integrated system has made the data collection become easier and more accurate than using a frequency generator. Also, it provides the portability of the design, availability to control speed of gas, better presentation to audience and easier operation in doing demonstration 
at various academic level.

Moreover, using different types of gas and tube diameter for Ruben's Tube show different results in speed of gas while inside the tube. This knowledge can be important in piping systems such as in oil and gas industries. Therefore, this review can be used to propose solutions and improvisations to show better a understanding of the properties of sound waves when there are changes to the type of gas used and tube diameter. The drawbacks discussed in this paper can be used to improve the future designs. Improving designs is important for education purposes at various academic level.

\section{ACKNOWLEDGEMENT}

This work is supported by Universiti Malaysia Sarawak (UNIMAS) under the Small Grant Scheme Project [F02/(S172)/1274/2015(09)] and Biasiswa Zamalah Unimas (ZSU).

\section{REFERENCES}

[1] Kent LG. The Rubens tube. In: Proceedings of Meetings on Acoustics, pp. 1-9; 2009.

[2] Heinrich R, Otto KM, Flammenrohre fur akustishe Beobachtungen. Annalen der Physik. 1905; 17:149-164.

[3] Sutton RM. Demonstration Experiments in Physics. McGraw Hill. 1938; 185.

[4] Halliday D, Resnick R. Physics, Wiley and Sons, New York. 1965; 504.

[5] Jihui D, Wang CTP. Demonstration of longitudinal standing waves in a pipe revisited. American Journal of Physics. 1985; 53: 1110-1112.

[6] Matt KF, Gregory EBJ, Jurgen FB, John WG. Experimental and numerical investigation of methane ignition and flame propagation in cylindrical tubes ranging from 5 to $71 \mathrm{~cm}$ - Part I: Effects of scaling from laboratory to large-scale field studies. Journal of Loss Prevention in the Process Industries. 2016; 41: 241251.

[7] Michael DG, Kent LG. An investigation of Rubens flame tube resonances. The Journal of the Acoustical Society of America, 2009; 125: 1285-1292.

[8] George PG, Jeffrey MB. Effect of external heat loss on the propagation and quenching of flames in small heat-recirculating tubes. Combustion and Flame, 2016; 173: 27-38.

[9] Ficken G, Stephenson C. Ruben's flame-tube demonstration. The Physics Teacher. 1979; 17: 306-310.

[10] Harold AD. A two-dimensional flame table. American Journal of Physics. 1987; 55: 733-737.

[11] Harold AD. The normal mode structure on the two-dimensional flame table. American Journal of Physics. 1988; 56: 913.

[12] Meiners HF. Physics demonstration experiments, New York: Ronald Press; 1970, p 315.

[13] Iona M. Pressure in standing waves. The Physics Teacher. 1976; 14: 325.

[14] Rossing TD. Average pressure in standing waves. The Physics Teacher. 1977; 15: 260.

[15] Jackson TL, Buckmaster J, Lu Z, Kyritsis DC, Massa L. Flames in narrow circular tubes. 2007; 31(1): 955-962. 
[16] Christophe A, Bruno D, Joel Q. Premixed flames propagating freely in tubes. Combustion and Flame, 2015; 4(162): 1225-1233.

[17] Brian EA, Brad M, Kent LG. Loudspeaker line array educational demonstration. 2014; 131(3): 2394-2400.

[18] Hailey A, Daniel R, Neil S. Testing the Speed of Sound in Various Gases Using a Ruben's Tube. University of Illinois at Urbana-Champaign, pp. 1-11; 2015.

[19] Selena B. A research about the effect of sound waves. In: Physics extended essay May 2009.

[20] Higuera FJ. Numerical simulation of the upward propagation of a flame in a vertical tube filled with a very lean mixture. Combustion and Flame, 2011; 5(158): 885-892.

[21] Simran M. Ruben's Tube: Determining the Effect of Frequency on Wavelength. In: Handbook and Proceedings of the Tennessee Junior Academy of Science, pp. 14-20; 2012.

[22] Coleman R. The flaming air track. The Physics Teacher 1975; 13: 556-557.

[23] Bauman RP, Moore D. More on dancing flames. The Physics Teacher 1977; 15: 448, 1977.

[24] Daisuke S, Yuuya H, Satoru I. Flame propagation in a vortex flow within small diameter tubes. In: Proceedings of the Combustion Institute, pp. 3251-3258, 2011.

[25] Todor T, Radhwan BM, Daniel B, Ricardo B, Vincent P. Firetrase: stereoscopic camera tracking and wireless wearable sensor system for interactive dance performances- application to fire experiences and projections. QPSR of the numediart research program 2010; 3(1): 9-24.

[26] Huahua X, Ryan WH, Elaine SO. Effects of pressure waves on the stability of flames propagating in tubes. In: Proceedings of the Combustion Institute, pp. 17, 2016.

[27] Yang J, Mossa FMS, Huang HW, Wang Q, Woolley R, Zhang Y. Oscillating flames in open tubes. In: Proceedings of the Combustion Institute, pp. 2075-2082; 2015.

[28] Chuan JZ, Zi SG, Bai QL, Zhong T, Yu MS, Qing Y, Yi DH, Chang G. Flame acceleration in pipes containing bends of different angles. Journal of Loss Prevention in the Process Industries 2016; 43: 273-279. 